\title{
Fast Particle Destabilization of Toroidicity induced Alfvén Eigenmodes in National Spherical Torus Experiment*
}

\author{
N. N. Gorelenkov, C. Z. Cheng, G. Y. Fu, S. Kaye, R. White \\ Princeton Plasma Physics Laboratory, P.O. Box 451 \\ Princeton, NJ 08543-0451 \\ M. V. Gorelenkova \\ TRINITI, Troitsk, Moscow region, 142092 Russia
}

(November 30, 1999)

\begin{abstract}
Toroidicity induced Alfvén Eigenmode (TAE) stability in National Spherical Torus Experiment (NSTX) is analyzed using the improved NOVA-K code, which includes finite orbit width and Larmor radiius effects and is able to predicts the saturation amplitude for the mode using the quasilinear theory. Broad spectrum of unstable global TAEs with different toroidal mode numbers is predicted. Due to the strong poloidal field and the presence of the magnetic well in NSTX better particle confinement in the presence of TAEs in comparison with tokamaks is illustrated making use of the ORBIT code.
\end{abstract}

\section{INTRODUCTION}

Since their theoretical predictions [1,2], toroidicity-induced Alfvén eigenmodes (TAE) were observed in many experiments on tokamaks including recent DT experiments on TFTR [3], in stellarators such as compact helical system (CHS) [4], and in spherical tokamak

\footnotetext{
*This work supported by DoE contract No. DE-AC02-76-CHO-3073.
} 
experiments such as START [5]. TAEs are believed to be able to cause a degradation of fusion product confinement in a reactor, and eventually terminating plasma burning.

The unique features of NSTX, such as low aspect ratio, high plasma and energetic particle beta, low Alfvén velocity with respect to beam ion injection velocity, and large Larmor radii present an entirely new regimes for studying energetic particle physics. In future experiments on NSTX two heating technics, Neutral Beam Injection (NBI) and High Harmonic Fast Wave Heating (HHFW), are proposed [6]. They may produce significant amount of super Alfvénic fast ions, which may induce a strong destabilizing drive for TAEs. For example, NBI ions will be tangentally injected at $\mathcal{E}=80 \mathrm{keV}$, which gives following estimates for the ratio of the injected ion velocity and the typical Alfvén velocity for the magnetic field $B=0.4 T$, and the plasma denisity $n_{e}=5 \times 10^{13} \mathrm{~cm}^{-3}: v_{b 0}=3 \times 10^{8} \mathrm{~cm} / \mathrm{s} \gg v_{A 0} \simeq 10^{8} \mathrm{~cm} / \mathrm{s}$. NBI ion Larmor radius will be $\rho_{L b} \simeq a / 4$, and drift orbit radial width $\Delta_{b}=(q / \epsilon) \rho_{L b} \simeq a / 2$. Note, that pevious START experiments [5] have shown strong TAE and chirping mode activity.

Despite the low aspect ratio and low magnetic field, i.e. large Larmor radius, single particle confinement is expected to be good in ST. This will be demonstrated in the fast particle loss calculations with and without TAEs.

In this paper TAEs in NSTX are analyzed using the improved NOVA-K code [8], which includes fast ion finite orbit width (FOW) and Larmor radius (FLR) effects to calculate the stability [9] and predicts the saturation amplitude for the mode using a quasilinear theory [10]. Then the ORBIT code is used [11] to evaluate the effect of TAEs on the confinement of fast neutral beam ions.

The paper is organized as follows. In Section II we present the results of the linear eigenmode study and mode stability using the perturbation technique. Results of TAE effects on fast particle population are given in Section III. The summary is presented in Section IV. 


\section{LINEAR EIGENMODE STUDY}

\section{A. Formulation}

In NOVA-K code we use a perturbative theory to calculate TAE growth rate [8]. The code is extended in Refs. [9], which containes the formulation accounting for particle FOW and FLR effects on TAE modes. In the following we outline the procedure.

Considering the fast particle contribution as a perturbation, one can obtain a quadratic form from the linearized momentum equation [8], which determines the TAE growth rate due to fast particle resonant interaction with oscillations

$$
\gamma_{h} / \omega \simeq \Im \delta W_{k} / 2 \delta K
$$

where $\delta K=\omega^{2} \int \rho|\xi|^{2} d r$ is the inertial energy, and $\delta W_{k}$ is the potential energy. The imaginary part of the hot particle contribution to the potential energy is determined by the following integral over the phase space

$$
\Im \delta W_{k}=i \frac{(2 M)^{2} \pi^{3} c}{z} \int d P_{\varphi} d \mu \frac{\sum_{m m^{\prime} l} G_{m^{\prime} l}^{*} G_{m l} \mathcal{E}^{5 / 2} \tau_{b} \sqrt{2} F_{\mathcal{E}}^{\prime}\left(\omega-\omega_{*}\right)}{\left|\left(\bar{\omega}_{D 0}+l \omega_{b}\right)_{v}^{\prime}\right|}
$$

where $P_{\varphi}$ is the toroidal canonical momentum, $\mu=v_{\perp}^{2} / 2 B$ is the particle magnetic moment per unit mass, $v$ is the particle velocity, $z$ is the particle charge, $\mathcal{E}$ is the particle energy, $F$ is the particle equilibrium distribution function, we denote ()$_{x}^{\prime}=\partial() / \partial x, \omega_{*}=$ $-n F_{P_{\varphi}}^{\prime} / F_{\mathcal{E}}^{\prime}, G_{m l}=\tau_{b}^{-1} \oint d t^{\prime} \hat{G}_{m} e^{i \int^{t^{\prime}}\left(\omega_{D m}-\omega\right) d t^{\prime \prime}}, \hat{G} \simeq \mathcal{E}^{-1}\left[(2 \mathcal{E}-3 \mu B) J_{0} k \xi_{\perp}-\mu B J_{0} \nabla \xi_{\perp}\right]$,

$\omega_{D m} \equiv m d \theta / d t-n d \varphi / d t$ is particle "drift" frequency and bar means orbit average, $\xi$ is plasma displacement vector, the integrand should be taken at the resonance velocity determined by the resonance condition $\omega-\bar{\omega}_{D 0}-l \omega_{b}=0$.

In NOVA code calculations the conservation of three integrals of particle unperturbed motion $v, \mu$, and $P_{\varphi}$ is assumed. This may not be true in the case of spherical tokamaks (ST), where typical vacuum field is low as a result of limitations on the central solenoid. In such conditions the hot ion gyroradius in the low field side can be as large as $20 \mathrm{~cm} \simeq a / 4$ for $80 \mathrm{keV}$ Deuterium ions and therefore $\mu$ may not be coserved, while $v$ and $P_{\varphi}$ are still 
conserved. Stochastic diffusion in $\mu$ is possible in this case and may result in isotropization of particle distribution function in the velocity space. and may be important for the NBI ions in NSTX. We note that the results of this paper may be still valid when the characteristic time of the diffusion in $\mu$ is less than the TAEs growth rate, but detailed study of this problem is beyond the scope of this paper.

\section{B. TAE structure.}

We analysed four NSTX equilibria covering a wide range of plasma parameters. First equilibrium has low central safety factor $q_{0}=0.4$, and at the edge $q_{e d g e}=15$, which corresponds to the TRANSP analysing code [12] run \#11112P60 with $\langle\beta\rangle \equiv 8 \pi\langle p\rangle /\left\langle B^{2}\right\rangle=10 \%$ $\left(\beta_{\text {tor }} \equiv 8 \pi\langle p\rangle / B_{\varphi 0}^{2}=34 \%\right)$. The second equilibrium has medium $q_{0}=0.7$, and $q_{e d g e}=16$ with $\langle\beta\rangle=10 \%$. The third and the fourth equilibria have high $q_{0}=2.8, q_{e d g e}=12$ with high beta $\langle\beta\rangle=15 \%$ and medium beta $\langle\beta\rangle=8 \%$, respectively. Pressure and density profiles are presented in the form $P(\psi)=P(0)\left(1-\psi^{1.03}\right)^{1.7}, n_{e}(\psi)=n_{e}(0)\left(1-\psi^{1.62}\right)^{0.48}$ for low- $q_{0}$ and medium- $q_{0}$ cases, while for high- $q_{0}$ case we use $P(\psi)=P(0)\left(1-\psi^{1.8}\right)^{2}$, $n_{e}(\psi)=n_{e}(0)\left(1-\psi^{10}\right)^{0.12}$. Vacuum magnetic field is $B_{0}=0.3 T$ at the geometrical axis. Figure 1 shows profiles in chosen equilibria. 


\section{FIGURES}
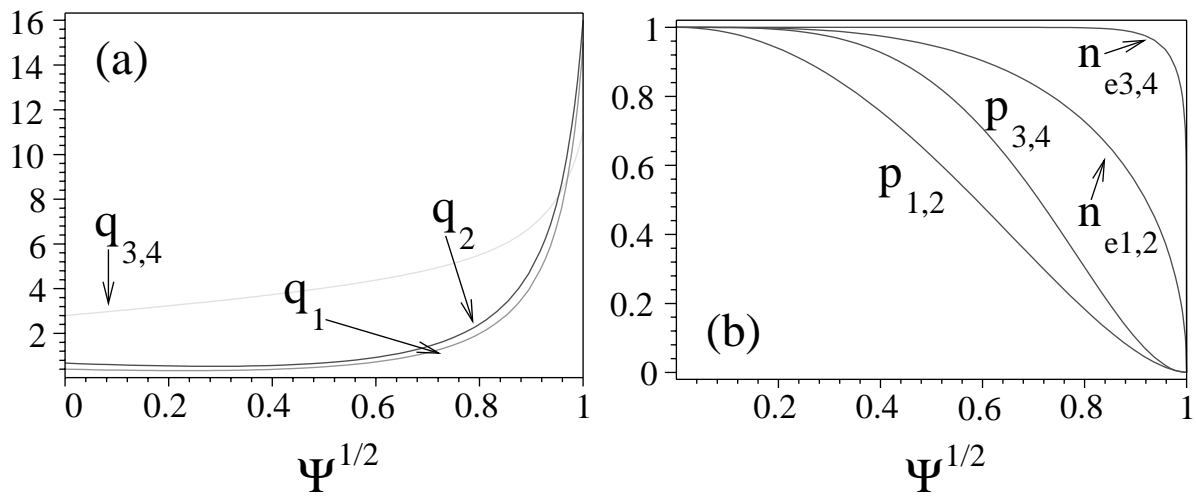

FIG. 1. Safety factor (a), and plasma pressure and density profiles (b) for four NSTX equilibria, where subscript corresponds to the number of the equilibrium.

Density and safety factor profiles are flat near the plasma center creating the aligned gap in the Alfvén continuum along the minor radius. Calculations show that the Alfvén gap is large due to the effect of strong toroidal coupling and does not close at high beta $\beta \simeq 1$, so that TAEs still can exist. Figure 2 illustrates the continuum gap structure, where the continuum frequency is normalized to the Alfvén frequency $\omega_{A}=v_{A 0} / q_{\text {edge }} R_{0}, R_{0}$ is the major radius of the geometrical center and $v_{A 0}$ is the Alfvén velocity evaluated with the central plasma mass density and vacuum magnetic field at the geometrical axis.

Fig.2(a) corresponds to a medium $q_{0}$ equilibrium with $\langle\beta\rangle=10 \%$ and Fig.2(b) corresponds to an equilibrium with increased beta $\langle\beta\rangle=33 \%$. One can see that the gap is open at higher pressure.
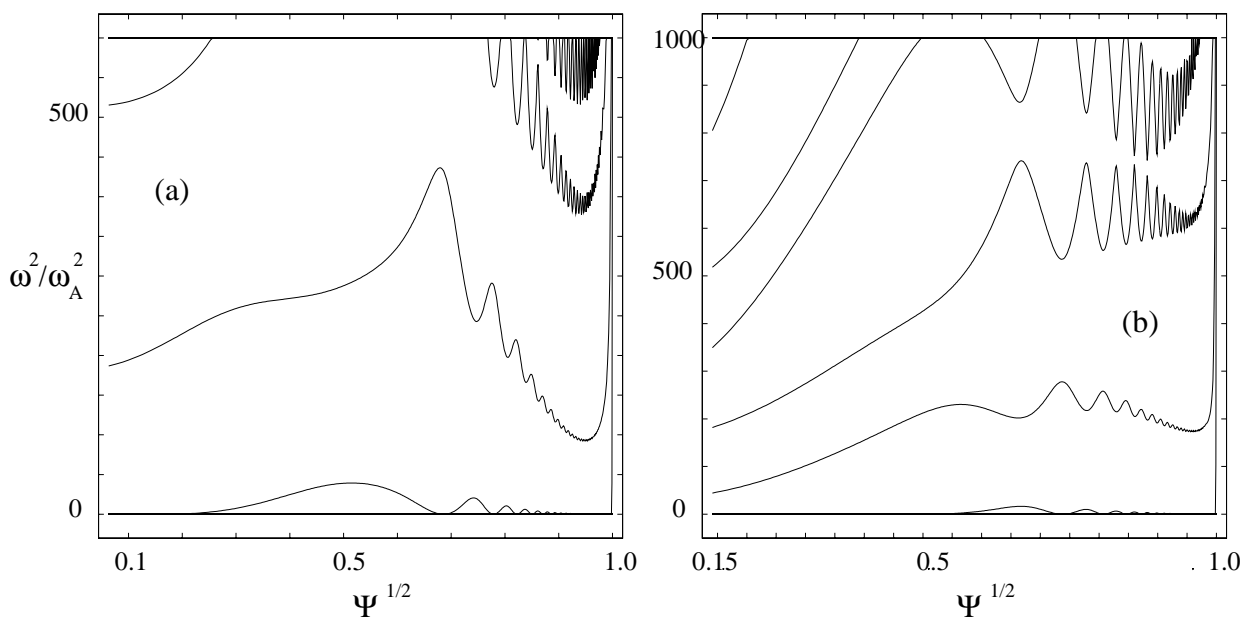
FIG. 2. Alfvén continuum gap in NSTX plasma for $n=3$ in medium- $q_{0}$ equilibrium with low $(<\beta>=10 \%)$ and high $(<\beta>=33 \%)$ plasma beta.

We note, that higher frequency gaps, i. e. gaps induced by noncircularity are usually closed in NSTX plasma, which means that it is harder to excite noncircularity induced Alfvén eigenmodes (NAE), then TAE.

For each toroidal mode number $n(n \leq 7)$, we found several TAE modes. Caclulations also show that in NSTX TAEs typically have very broad radial envelop structure covering the whole minor radius. Ions may be super-Alfvénic if their energy satisfies $\mathcal{E}>>K \backslash \mathcal{V}$. This indicates that plasma ion $\omega_{* i}$ effects may be important but are neglected in our model.

We performed calculations of TAE modes with toroidal mode numbers from low to medium $n=1,3,5,7$. Going to higher $n$ is limited by the computational limitations as required number of poloidal harmonics need to be equal roughly to $n q(a)$, which is rather high for NSTX with typically $q(a)>10$. A number of TAE modes were found: 6 for the low- $q_{0}$ equilibrium, 22 for the medium- $q_{0}$ equilibrium, 11 for the high- $q_{0}$ high- $\beta$ equilibrium, and 8 for the high- $q_{0}$ medium- $\beta$ equilibrium. Examples of two TAE mode structure are shown in Figure 3
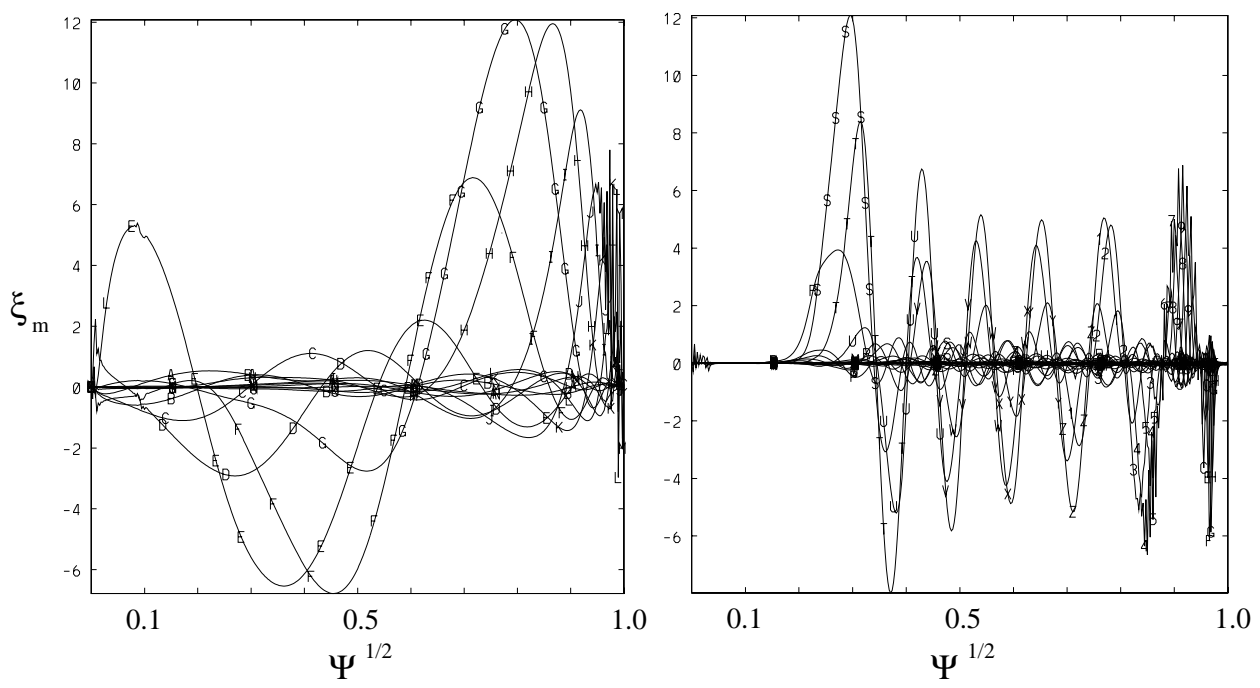

FIG. 3. Most unstable TAEs structure for $n=1$ and $n=5$ in high beta plasma at $q_{0}=2.8$.

and they were found to be the most unstable. The poloidal mode numbers for each 
harmonic are labeled according to the notations: 'A' corresponds to $m=-1$, 'B' is $m=0$, and ' $\mathrm{C}$ ' is $m=1$ and so on.

\section{TAE stability.}

Our analysis of TAE stability in NSTX is perturbative with eigenmode structure calculated by the ideal MHD code NOVA [7] [8] and with drive and damping calculated by the postprocessor code NOVA-K [8] [9]. Fast particle drive inlcudes FOW and FLR effects and is computed according to Eq.(1). NOVA-K code was recently improved [9] to analyse the stability of Alfvén modes with arbitrary particle orbit width in general tokamak geometry. Trapped electron collisional damping is modified and includes now not only trapped electron interaction with parallel electric field [13], but also electron compression effect as well.

TAEs are driven by the pressure gradient of NBI ions, which are injected tangentially to the major radius with beam width roughly equal to one half of the minor radius. The distribution function of fast ions needs to be calculated separately and is beyond the scope of this work, so that we assume the distribution function of fast ions to be slowing down in energy with a gaussian distribution in pitch angle $\lambda=\mu B_{0} / E$, which is peaked at $\lambda=0.3$ and has width $\Delta \lambda=0.5$. Fast particle radial pressure profile is given by TRANSP for lowand medium- $q_{0}$ equilibriums and is $P_{b}(\psi)=P_{b 0}\left(1-\psi^{1.33}\right)^{3.4}$ for high- $q_{0}$.

Table I summarizes the results of the stability study for $n=1,3,5,7$ modes. 


\section{TABLES}

\begin{tabular}{|c|c|c|r|r|r|r|}
\hline \hline equilibrium & $\langle\beta\rangle, \%$ & $\beta_{b}(0), \%$ & stable & unstable & of & modes with \\
modes & modes & $\gamma / \omega_{A}>30 \%$ & $\beta_{b c r i t}(0), \%$ \\
\hline low- $q_{0}$ & 10 & 63 & 6 & 0 & 0 & 90 \\
\hline medium- $q_{0}$ & 10 & 11 & 19 & 3 & 0 & 9 \\
\hline high- $q_{0}$ & 15 & $>22$ & 6 & 5 & 6 & $0(1)$ \\
\hline high- $q_{0}$ & 8 & $>10$ & 5 & 3 & 2 & $0(15)$ \\
\hline \hline
\end{tabular}

TABLE I. TAE stability analysis statistics and lowest fast ion critical beta for $n=1,3,5,7$ modes. 
It shows the values of fast particle central beta, the number of stable and unstable eigenmodes for a given equilibrium, and the lowest critical fast particle beta above which the mode is unstable. In NSTX linear perturbative calculations predict very strong drive for TAEs, which may be as high as $\gamma / \omega>30 \%$ and makes our perturbative approach inaccurate (but not to change the qualitative stability properties). The eigenmode structure and the eigenfrequency can be modified as compared to the MHD solution by fast particles during the mode nonlinear evolution. Other important result of our calculations is that NOVA-K predicts unstable modes for higher $n(n>3)$ modes even without fast particles, but with the same thermal plasma parameters to ensure the same plasma equilibrium. The drive is because $\omega<\omega_{* i}$ and results from the tail of the Maxwellian plasma ions, which at energies $\mathcal{E} \simeq 6 k e V$ have the velocity close to the Alfvén velocity. For such case $(n=5)$ the value of the critical beta of fast ions is zero as shown in the table. The paranthesis shows the lowest critical beam beta for the modes with thermal ion damping $(n \leq 3)$. The dominant damping mechanism is the ion Landau damping for all the cases studied.

\section{TAES EFFECTS ON FAST ION TRANSPORT}

The guiding center orbit code ORBIT [11] is used to calculate the efect of TAEs on the fast particle confinement in NSTX. Because of strong poloidal harmonic coupling each eigenmode has to be represented by many poloidal harmonics, which is time consuming procedure for particle codes such as ORBIT. Thus, we consider $n=1$ TAE with the highest drive for each equilibrium. For the analysis of two unstable TAEs we choose $n=1$ and $n=3$. NOVA-K is capable of predicting TAE's amplitude using the quasilinear theory [9] [10], and can be used to evaluate the TAE amplitude in NSTX plasma. For the purpose of this paper we consider only the trend in the parametric dependence of the eigenmode apmlitude. We found that typically the predicted mode amplitude is at least an order magnitude higher in NSTX than in TFTR for the same growth rate to the damping rate ratio. This is due

to higher "effective" collisionality in NSTX. Basing on NOVA-K calculations we choose the 
TAE amplitude to have the same value for all eigenmodes with $\tilde{B}_{\theta} / B=10^{-3}$. Table III shows the results of ORBIT calculation of beam ion loss fraction for low $q_{0}=0.4,\langle\beta\rangle=10 \%$ and high $q_{0}=2.8,\langle\beta\rangle=15 \%$ equilibria. Shown are total prompt losses which occur when no mode is present and the losses mostly happen during the first particle transit over the drift orbit. Also shown are total fast ion losses when one or two modes are included.

\begin{tabular}{|c|c|c||c|c|c|}
\hline- & low- $q_{0}$ & - & - & high- $q_{0}$ & - \\
\hline losses, $\% \rightarrow$ & prompt & $n=1$ TAE & prompt & $n=1$ & $n=1 \& n=3$ \\
\hline no FLR & 9 & 11 & 1 & 2 & \\
\hline with FLR & 29 & 31 & 24 & 30 & 35 \\
\hline
\end{tabular}

TABLE III. Particle losses as simulated by ORBIT in low- and high- $q_{0}$ equilibria 
In tokamaks at such TAE amplitudes resonances usually overlaps and produce significant particle losses [14] [15]. In NSTX dispite the large beam ion Larmor radii the strong edge poloidal magnetic field helps to confine particle at high beta, so that TAEs do not produce large additional losses. Note, we included FLR effect in the ORBIT code only approximatly. At each step on a particle guiding center trajectory the Larmor radius $\rho_{h}$ is evaluated. If a particle comes to the last flux surface within $\rho_{h}$ distance it is considered lost. This may overestimate the losses because the Larmor radius may be smaller at the edge and the poloidal field is stronger than toroidal at the low field side of the last flux surface.

Poloidal magnetic field is strong only at the edge, so that one can expect particle redistribution mostly inside the plasma. This is seen from Fig.4, which shows the radial distribution of NBI ions without and with TAE. The TAE mode has $n=1$ and is the same mode used in previous particle loss calculations. The equilibrium has a high $q_{0}=2.8$ and $\langle\beta\rangle=15 \%$.
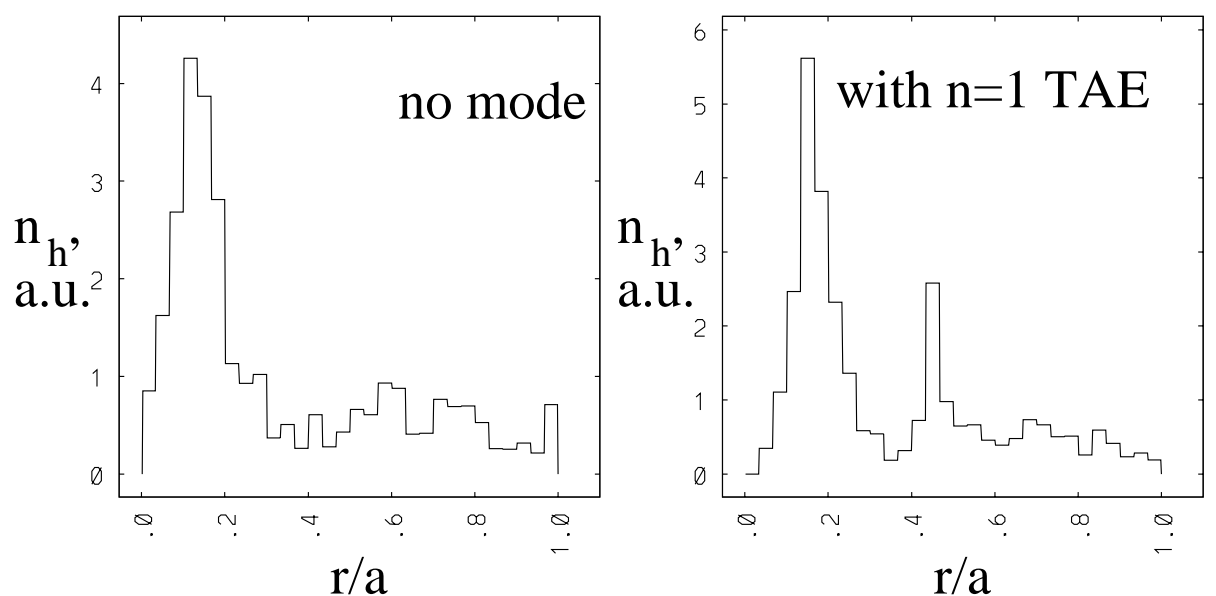

FIG. 4. NBI ions before and after the interaction with $n=1$ TAE at the perturbation amplitude $\tilde{B}_{\theta} / B=10^{-3}$.

Similar internal redistribution was observed when two modes are present.

\section{SUMMARY}

Many TAEs may be unstable in NSTX. TAEs are found having global radial structure. Alfvén continuum gap exist even at very high plasma beta, with TAE modes present. TAEs may have strong drive $\gamma / \omega>30 \%$, which requires nonperturbative codes for more accurate 
calculations. Single and two mode calculations predict highest beam ion losses totaling in high beta high- $q_{0}$ plasmas $\sim 35 \%$ of the NBI ion population with FLR effects included, where most of the losses are prompt losses (24\%), with TAE effects resulting in additional loss of $11 \%$ of NBI ion population. Good fast ion confinement is observed in high beta plasmas because of the strong poloidal field at the edge.

NOVA-K also produces unstable TAE solution in START experiments with $n=1$ [5] in case of low beta plasma. In high beta plasma the chirping modes were also observed in START, which usually indicates strong drive of the mode or even creating a new resonant

branch of TAEs [16]. To provide the understanding of TAE drive and damping in ST further analysis is needed.

\section{ACKNOWLEDGMENT}

This work was supported by US DoE contract DE-AC02-76-CHO-3073. 


\section{REFERENCES}

[1] C. Z. Cheng, L. Chen, and M. S. Chance, Ann. Phys. (N.Y.) 161, 21 (1985).

[2] C. Z. Cheng, and M. S. Chance, Phys. Fluids B 161, 3695 (1986).

[3] R. Nazikian, et.al., Phys. Plasmas, 51703 (1998).

[4] M. Takechi, K. Toi, S. Takagi, et.al. Phys. Rev. Lett. 83312 (1999).

[5] K. G. McClements, et.al., Plasma Phys. and Control. Fusion 41, 661 (1999).

[6] S. M. Kaye, M. Ono, Y-K. M. Peng, et.al. Fusion Technology 3616 (1999).

[7] C. Z. Cheng, and M. S. Chance, J. Comput. Phys. 71, 124 (1987).

[8] C. Z. Cheng, Phys. Reports 2111 (1992).

[9] N. N. Gorelenkov, C. Z. Cheng, and G. Y. Fu, Phys. Plasmas 62802 (1999).

[10] N. N. Gorelenkov, et.al., Phys. Plasmas 6629 (1999).

[11] R. B. White, M. S. Chance, Phys. Fluids 272455 (1984).

[12] R. V. Budny, et. al., Phys. Plasmas 3, 4583 (1996).

[13] G. Y. Fu, et. al., Phys. Fluids 54040 (1993).

[14] C. T. Hsu, D. J. Sigmar, Phys. Fluids B 41492 (1992).

[15] C. Z. Cheng, R. V. Budny, L. Chen, et.al., in Plasma Physics and Controlled Nuclear Fusion Research 1994: Proceedings of the 15th IAEA Conference Seville (IAEA, Viena, 1995) 3373.

[16] C. Z. Cheng, N. N. Gorelenkov, and C. T. Hsu, Nucl. Fusion 35, 1639 (1995). 